Research Article

\title{
Transcriptional profile of fibroblasts obtained from the primary site, lymph node and bone marrow of breast cancer patients
}

\author{
Paulo Roberto Del Valle ${ }^{1}$, Cintia Milani ${ }^{1}$, Maria Mitzi Brentani ${ }^{1}$, Maria Lucia Hirata Katayama ${ }^{1}$, \\ Eduardo Carneiro de Lyra ${ }^{2}$, Dirce Maria Carraro ${ }^{3}$, Helena Brentani ${ }^{4}$, Renato Puga ${ }^{3}$, Leandro A. Lima ${ }^{3}$, \\ Patricia Bortman Rozenchan ${ }^{1}$, Bárbara dos Santos Nunes ${ }^{1}$, João Carlos Guedes Sampaio Góes ${ }^{2}$ \\ and Maria Aparecida Azevedo Koike Folgueira ${ }^{1}$ \\ ${ }^{1}$ Departamento de Radiologia e Oncologia, Faculdade de Medicina, Universidade de São Paulo, \\ São Paulo, SP, Brazil. \\ ${ }_{2}^{2}$ Instituto Brasileiro do Controle do Câncer, São Paulo, SP, Brazil. \\ ${ }^{3}$ Centro Internacional de Pesquisa e Ensino, Hospital A.C. Camargo, São Paulo, SP, Brazil. \\ ${ }^{4}$ Departamento de Psiquiatria, Faculdade de Medicina, Universidade de São Paulo, São Paulo, SP, Brazil.
}

\begin{abstract}
Cancer-associated fibroblasts (CAF) influence tumor development at primary as well as in metastatic sites, but there have been no direct comparisons of the transcriptional profiles of stromal cells from different tumor sites. In this study, we used customized cDNA microarrays to compare the gene expression profile of stromal cells from primary tumor $(\mathrm{CAF}, \mathrm{n}=4)$, lymph node metastasis $(\mathrm{N}+, \mathrm{n}=3)$ and bone marrow $(B M, n=4)$ obtained from breast cancer patients. Biological validation was done in another 16 samples by RT-qPCR. Differences between CAF vs $\mathrm{N}+$, CAF vs $\mathrm{BM}$ and $\mathrm{N}+$ vs BM were represented by 20, 235 and 245 genes, respectively (SAM test, FDR < 0.01). Functional analysis revealed that genes related to development and morphogenesis were overrepresented. In a biological validation set, NOTCH2 was confirmed to be more expressed in $\mathrm{N}+$ (vs CAF) and ADCY2, HECTD1, HNMT, LOX, MACF1, SLC1A3 and USP16 more expressed in BM (vs CAF). Only small differences were observed in the transcriptional profiles of fibroblasts from the primary tumor and lymph node of breast cancer patients, whereas greater differences were observed between bone marrow stromal cells and the other two sites. These differences may reflect the activities of distinct differentiation programs.
\end{abstract}

Keywords: bone marrow, breast neoplasm, fibroblast, gene expression profiling, lymph node, mesenchymal stromal cells, tumor microenvironment.

Received: December 16, 2013; Accepted: April 19, 2014.

\section{Introduction}

Fibroblasts are mesenchymal cells derived from the mesoderm and are involved in maintaining tissue architecture. These cells secrete a variety of soluble factors that modulate surrounding cell functions.

Cancer-associated fibroblasts (CAF) are the most prominent cell type in the stroma of primary tumors. These CAFs are qualitatively different from normal fibroblasts (Orimo et al., 2005) and heterotypic interactions between cancer cells and stromal cells lead to malignant cell proliferation and metastasis. Indeed, the loss of tumor suppressor genes such as PTEN (Trimboli et al., 2009) or TP53 (Patocs et al., 2007) in stromal fibroblasts results in the activation

Send correspondence to Maria Aparecida A. Koike Folgueira. Departamento de Radiologia e Oncologia, Faculdade de Medicina, Universidade de São Paulo, Avenida Dr. Arnaldo 455, 4ำ andar, sala 4124, 01246-903 São Paulo, SP, Brazil. E-mail: makoike@lim24.fm.usp.br. of pathways leading to epithelial cell carcinogenesis or regional node involvement.

The impact of CAFs on patient prognosis provides insight into their tumor-specific effects (Paulsson and Micke, 2014). The total fibroblast content of breast tumors and the tumor-stroma ratio $(<50 \%$ vs. $>50 \%$ stroma $)$ have an independent impact on recurrence-free and overall survival (de Kruijf, 2011). A higher proportion of $\alpha$ SMA myofibroblasts in breast tumors is also associated with higher proliferation of tumor cells and an unfavorable prognosis (Surowiak et al., 2007). In addition, a metabolic partnership between catabolic fibroblasts and anabolic cancer cells creates a nutrient-rich environment, even under hypoxia, that supports tumor growth (Martinez-Outschoorn et al., 2014).

Several hypotheses have tried to establish the origin of CAFs. First, it is well known that resident fibroblasts, when in contact with malignant cells, become activated myofibroblasts that express specific markers such $\alpha$ SMA 
(alpha smooth muscle actin) (Kalluri and Zeisberg, 2006). Second, epithelial or endothelial cells can trans-differentiate to acquire a mesenchymal phenotype in a process known as epithelial or endothelial mesenchymal transition (Petersen et al., 2003; Zeisberg et al., 2007; Guarino et al., 2009). Third, mesenchymal stem cells (derived from bone marrow or adipose tissue) are recruited to the tumor site to create a favorable microenvironment, in a process similar to wound healing, thereby giving rise to $20-40 \%$ of CAFs (Karnoub et al., 2007; Mishra et al., 2008; Shimoda et al., 2010; Quante et al., 2011).

In breast cancer, the involvement of regional lymph nodes is associated with poor prognosis (Kim et al., 2006; Edge and Compton, 2010). Normal lymph nodes contain specific fibroblasts known as reticular cells that are specialized in maintenance of the nodes reticular fiber architecture and the maturation of lymphocytes that express specific chemokines (Vega et al., 2006). However, little is known about the role of fibroblasts in lymph node metastasis. In accordance with the primary tumor site, involved lymph nodes show an altered microenvironment that includes the presence of activated fibroblasts (Yeung et al., 2013). In addition, lymph node fibroblasts may also induce the proliferation and migration of breast cancer cells (Santos et al., 2011).

The origin of fibroblasts in metastatic lymph nodes is speculative, but one possibility is that they originate from the same sources hypothesized for the primary tumor site. Moreover, recent observations suggest that cancer cells carry fibroblasts during their migration to metastatic sites such that these co-traveling cells may facilitate tumor progression in secondary organs (Duda et al., 2010).

Bone marrow $(\mathrm{BM})$ is a common homing organ and a reservoir for disseminated tumor cells in breast cancer (BC). Although only a small fraction of patients present overt BM metastasis, $70 \%$ of advanced breast cancer patients develop bone metastasis. Bone marrow is a source of mesenchymal stem cells (MSc) that can give rise to cells of mesodermal lineages (El-Haibi and Karnoub, 2010) such as adipocytes, osteocytes and chondrocytes. Besides playing an important pro-tumorigenic role in the microenvironment of bone metastasis (Martinez et al., 2013), these mesenchymal stem cells can also home to sites of tumorigenesis and integrate into the tumor stroma (El-Haibi and Karnoub, 2010).

It is still unclear whether the transcriptional profile can reveal the cellular origin of CAFs. Given that bone marrow stromal cells are a candidate progenitor cell source, the main goal of this work was to compare the gene expression profiles of stromal cells from the primary tumor, lymph nodes and bone marrow of breast cancer patients.

\section{Material and Methods}

\section{Patients}

This study was done in accordance with the Declaration of Helsinki (1964) and was approved by the Institu- tional Ethics Committees (Comitê de Ética do Hospital das Clínicas da Faculdade de Medicina da Universidade de São Paulo, CAPPesq process nos. 110/02, 368/03, 102/05, 0848/08 and 055/11; and Comitê de Ética do Instituto Brasileiro do Controle do Câncer; approved on 08/02/2002 and 07/27/2008). Twenty-seven breast cancer patients provided signed informed consent and were enrolled in the study. Samples from 11 patients were destined for microarray experiments and from 16 patients were used in biological validation studies. Primary tumor, lymph node metastasis and bone marrow samples were obtained from 11,8 and 8 patients, respectively (Table 1 ). The patients received no neoadjuvant chemotherapy or radiotherapy prior to sample collection. All patients were treated at the Instituto Brasileiro de Controle do Câncer (IBCC) in São Paulo city, Brazil.

\section{Primary cell culture}

All tumor samples (primary tumor, lymph node and bone marrow) were collected during breast surgery. Involvement of the primary site and lymph node was confirmed histologically. The samples were cut into small pieces and fibroblast primary cultures were established through explant methodology. The mesenchymal origin of the cells was confirmed by the expression of vimentin and lack of expression of pan-cytokeratin and CD45. The culture conditions were as previously described (Rozenchan et al., 2009; Santos et al., 2011; Campos et al., 2013). aSMA expression was detected in all samples from primary tumor and lymph nodes and has been described in previous reports (Rozenchan et al., 2009; Santos et al., 2011; Campos et al., 2013).

Bone marrow aspirates from the sternal area were collected during the breast surgical procedure. Stromal cells were recovered from the mononuclear cell layer by using a Ficoll-Paque ${ }^{\mathrm{TM}}$ Plus solution gradient (Amersham Biosciences, Amersham, England) (Sekiya et al., 2002) and plated in $75 \mathrm{~cm}^{2}$ culture flasks. The cultures were maintained in Iscove's Modified Dulbecco's Medium (IMDM) (Gibco, New York, USA), supplemented with HEPES (25 $\mathrm{mM})$, ampicillin $(100 \mu \mathrm{g} / \mathrm{mL})$, streptomycin $(100 \mu \mathrm{g} / \mathrm{mL}$ ) and $10 \%$ fetal bovine serum (Gibco). Onehalf of the supernatant with non-adherent cells was removed weekly and replaced with fresh medium until a confluent layer was obtained. After three cell passages, morphologically homogeneous cultures containing only fibroblastoid cells were obtained and used for further studies. The mesenchymal origin was confirmed by the expression of human vimentin (clone Vim 3b4, 1:100; Dako Corporation, Carpinteria, USA) and lack of expression of CD-45 (clone 2B11/PD7/26, 1:500; Dako) and cytokeratin (AE1/AE3, 1:50; Dako).

\section{Detection of occult tumor cells}

Occult tumor cells were detected in bone marrow aspirates via nested-PCR that detected the expression of cyto- 
Table 1 - Patients characteristics.

\begin{tabular}{|c|c|c|c|c|c|c|c|}
\hline Sample & Age & Clinical stage & Histological type & Lymph node & ER & PR & ErbB2 \\
\hline *CAF_1 & 52 & II & IDC & - & - & - & + \\
\hline *CAF_2 & 44 & II & IDC & - & - & + & - \\
\hline *CAF_3 & 53 & II & ILC & - & - & - & - \\
\hline * CAF_4 & 79 & II & IDC & - & + & + & - \\
\hline$* \mathrm{~N}+{ }_{-} 1$ & 59 & III & ILC & + & - & + & - \\
\hline$* \mathrm{~N}+22$ & 41 & III & IDC & + & - & + & - \\
\hline$* \mathrm{~N}+{ }_{-} 3$ & 49 & III & IDC & + & + & + & + \\
\hline *BMMC_1 & 54 & I & IDC & - & + & + & + \\
\hline * BMMC_2 & 69 & I & IDC & - & + & + & - \\
\hline *BMMC_3 & 76 & III & IDC & + & + & + & - \\
\hline * BMMC_4 & 57 & II & IDC & + & + & + & - \\
\hline$\dagger \mathrm{CAF} \_5$ & 57 & III & IDC & + & + & + & + \\
\hline$\dagger$ CAF_6 & 63 & III & IDC & + & ND & ND & ND \\
\hline$\dagger \mathrm{CAF}_{-} 7$ & 50 & I & IDC & - & + & + & - \\
\hline$\dagger \mathrm{CAF} \_8$ & 66 & II & IDC & + & + & + & + \\
\hline$\dagger$ CAF_9 & 57 & II & IDC & - & + & + & + \\
\hline$\dagger$ CAF_10 & 41 & II & IDC & - & + & + & + \\
\hline$\dagger \mathrm{CAF} \_11$ & 82 & III & IDC & + & - & - & + \\
\hline$\dagger \mathrm{N}+\_4$ & 47 & II & IDC & + & + & + & + \\
\hline$\dagger \mathrm{N}+55$ & 40 & II & IDC & + & + & + & - \\
\hline$\dagger \mathrm{N}+6$ & 65 & II & IDC & + & - & - & - \\
\hline$\dagger \mathrm{N}+\_7$ & 81 & III & IDC & + & + & + & - \\
\hline$\dagger \mathrm{N}+8$ & 43 & II & ILC & + & $\mathrm{ND}$ & ND & ND \\
\hline$\dagger$ †MMC_5 & 51 & III & IDC & + & + & + & - \\
\hline † BMMC_6 & 63 & II & IDC & - & - & - & + \\
\hline$\dagger$ BMMC_7 & 41 & III & IDC & + & + & + & - \\
\hline$\dagger$ †MMC_8 & 50 & II & IDC & - & + & + & - \\
\hline
\end{tabular}

*Samples used in microarray experiments. †Samples used in biological validation set in RT-qPCR experiments. CAF - fibroblasts from primary tumor; $\mathrm{N}+$ - fibroblasts from lymph node; BMMC - bone marrow mesenchymal cells. IDC - invasive ductal carcinoma; ILC - invasive lobular carcinoma; $(+)$ positive or (-) negative immune-expression for ER - estrogen receptor; PR - progesterone receptor. ND - not determined.

keratin 19 (KRT19) in cDNA samples (Table S1). The sensitivity was determined by serially diluting MDAMB231 cells that express KRT19 in peripheral blood mononuclear cells from a healthy donor; with this approach it was possible to detect one epithelial tumor cell in a million. An external amplicon of 798 bp was generated by 35 cycles in the first reaction of the nested-PCR. The product of the first reaction was then subjected to a second amplification of 30 cycles that generated a final product of $461 \mathrm{bp}$ in bone marrow aspirates containing occult tumor cells.

\section{RNA extraction and reverse transcriptase reaction}

Total RNA was extracted using TRIZOL ${ }^{\circledR}$ reagent (Invitrogen); purity was assessed by measuring the absorbance at 260 and $280 \mathrm{~nm}(260 / 280$ ratio > 1.7) and intactness was assessed by visualizing the $28 \mathrm{~S}$ and $18 \mathrm{~S}$ bands $(28 \mathrm{~S} / 18 \mathrm{~S}$ ratio $>1.5)$ after electrophoresis in $1.5 \%$ agarose gels. cDNAs for microarray validation were reverse tran- scribed from $2 \mu \mathrm{g}$ of total RNA in a $20 \mu \mathrm{L}$ reaction, using $2 \mu \mathrm{L}$ of random hexamers $(0.5 \mu \mathrm{g} / \mu \mathrm{L})$ and $200 \mathrm{U}$ of reverse transcriptase (Superscript II, Invitrogen).

\section{cDNA microarray assembly, hybridization and analysis}

Total RNA from stromal cell primary cultures was processed through a two-round RNA amplification procedure done by combining antisense RNA (aRNA) amplification with a template-switching effect, according to a previously described protocol (Wang et al., 2000; Baugh et $a l ., 2001)$. Three to five micrograms of aRNA was then used in a reverse transcriptase reaction in the presence of Cy3- or Cy5-labeled dCTP (GE Heathcare, Piscataway, NJ, USA) and SuperScript II (Invitrogen). The Hb4A normal epithelial mammary cell line was used as a reference. Hybridizations were done on customized cDNA microarray 
slides containing 4608 human genes (Brentani et al., 2005) (GEO accession number GPL1930) in a GeneTac Hybridization Station (Genomic Solutions). Dye swap was done for each sample analyzed to control for dye bias. Hybridization was done at $65^{\circ} \mathrm{C}$. Hybridized arrays were scanned with a confocal laser scanner (Arrayexpress, PerkinElmer Life and Analytical Science Inc., Boston, MA, USA) and the data were recovered with Quantarray software (PerkinElmer). Quantified signals were then submitted to $\log$ transformation and to Lowess normalization.

\section{Microarray data analysis}

To identify differences among stromal cells from primary tumor, lymph node and bone marrow, data obtained from the three sites were normalized together and only genes present in $100 \%$ of the samples were submitted to SAM (significance analysis of microarray) multiclass statistical test using FDR $<0.01$. Subsequently, to identify specific differences, differentially expressed genes were further analyzed with the SAM two class test, with FDR $<0.01$. Hierarchical clustering based on Euclidian distances and complete linkage was done using the differentially expressed genes, with reliability assessed by the bootstrap technique (TMEV software). Functional analysis was done using the DAVID (Database for Annotation, Visualization and Integrated Discovery) v.6.7 and IPA (Ingenuity Pathway Analysis ${ }^{\mathcal{O}}$, Ingenuity Systems, Inc.). To identify differences in bone marrow according to the presence of occult cells, we used the HT self-self statistical test for low sample numbers (Vêncio and Koide, 2005).

\section{RT-qPCR}

Primer sets were designed using Primer Blast (Table S1). Sequences present in different exons were selected. Quantitative RT-PCR was done using a Power Sybr Green PCR master mix (Applied Biosystems) in an ABI HT 7900 thermo cycler (Applied Biosystems). All samples were tested in duplicate and average values were used for quantification. Relative expression was calculated by the $2^{-\Delta \Delta \mathrm{Ct}}$ method (Livak and Schmittgen, 2001). The gene PPIA was selected as a reference gene and the Ct average (for the same transcript) of all samples was used as a reference.

\section{Results}

\section{Patients}

Twenty-seven patients provided written informed consent and were enrolled in the study. Patient age ranged from 40 to 82 years (median: 54 years). According to the clinical stage, the majority (17 patients) were stage II, ten patients were stage III and three were stage I. Sixteen patients had lymph node metastasis at surgery. Immunohistochemical evaluation of the tumors indicated that five patients were negative for hormonal receptors (estrogen or progesterone receptors), whereas 20 patients were positive for at least one hormonal receptor, 15 patients were negative and ten patients were positive for the ErbB2 receptor; it was not possible to evaluate the receptor status of two patients (Table 1).

\section{Influence of occult tumor cells in primary cultures of bone marrow mesenchymal cells}

As bone marrow may be a homing organ and a reservoir for disseminated tumor cells in breast cancer, we used nested-PCR to screen bone marrow aspirates for cytokeratin 19 expression, a marker of occult tumor cells. Among eight patients who had bone marrow aspirates collected during surgery, three tested positive for cytokeratin 19 expression (BMMC_3, BMMC_4, BMMC_6, Table 1).

We then examined whether bone marrow mesenchymal cell gene expression was associated with the presence of occult malignant cells in bone marrow. After establishing primary cultures, samples from two patients with lymph node involvement and the presence of occult tumor cells in bone marrow and samples from two patients with no lymph node involvement and a lack of occult tumor cells were selected. Twenty-one differentially expressed genes with a 1.38- to 7.20-fold change in expression were identified and included nine upregulated (PTHLH, TLOC1, NCOA6, C17orf57, ANAPC11, MAST4, POLR3E, CPNE1 and B4GALT5) and 12 downregulated (MRPL2, NAT10, DAP, RNF2, FLOT2, FKBP10, SLIT3, EBNA1BP2, SLC35B2, MICAL2, GPR3 and TSPAN17) genes in bone marrow mesenchymal cells from patients with occult tumor cells. However, none of the selected genes were confirmed as differentially expressed when evaluated by RT-qPCR (data not shown). These results indicate that the presence of occult malignant cells does not significantly affect the molecular characteristics of bone marrow stromal cells.

\section{Gene expression profile of mesenchymal cells from bone marrow, primary tumor and lymph node}

Gene expression was initially evaluated using all of the genes represented in the microarray. Comparison of the expression profile of stromal cells from the primary tumor, lymph node and bone marrow of breast cancer patients identified 296 genes that were differentially expressed (SAM multiclass test) (Figure S1). However, since some samples had genes that gave no signal (probably because of methodological errors rather than biological issues) we excluded these genes and reanalyzed the samples. This reevaluation included only genes present in all of the samples and revealed 267 differentially expressed genes among fibroblasts from different origins. (Table S2, Figure 1A). These genes clustered the samples according to their origin, indicating that differences reflecting the samples origin were more important than differences among individual patients (Figure 1B). To detect specific differences related to 
the cell origin, a second analysis was done by comparing two groups at a time using the SAM two-class test.

Comparison of fibroblasts obtained from the primary tumor and lymph node identified 20 differentially expressed genes (eight less expressed and 12 more expressed in the primary tumor). When fibroblasts from the primary tumor and bone marrow were compared, 235 differentially expressed genes were observed (101 less expressed and 134 more expressed in bone marrow), and when fibroblasts from lymph node and bone marrow were compared, 245 differentially expressed genes were identified (105 less expressed and 140 more expressed in bone marrow stromal cells) (Table S2). These findings showed that (1) the gene expression profiles of CAFs and $\mathrm{N}+$ were quite similar, (2) the majority of genes were differentially expressed in comparisons of stromal cells from each of these two tumor sites (CAFs and $\mathrm{N}+$ ) with bone marrow mesenchymal cells, and (3) bone marrow stromal cells had the most differential profile among the three sites (Table S2).

\section{Functional annotation and selection of candidate genes}

An important issue when analyzing gene lists is to detect enriched functions. For this analysis, the 267 genes differentially expressed among bone marrow mesenchymal cells, CAFs and $\mathrm{N}+$, were grouped as more expressed in bone marrow stromal cells and more expressed in CAFs and $\mathrm{N}+$. These genes were then used as input data and biological processes from Gene Ontology were screened for enrichment. Functions enriched among genes less expressed in bone marrow mesenchymal cells (and more expressed in $\mathrm{CAFs}$ and $\mathrm{N}+$ ) included negative regulation of nucleobase, nucleoside, nucleotide and nucleic acid metabolic processes, negative regulation of transcription and apoptosis (Table S3) and functions enriched among genes more expressed in bone marrow mesenchymal cells (as compared with CAFs or $\mathrm{N}+$ ) included mesoderm formation, response to wounding, regeneration and embryonic morphogenesis, with the latter including only a few genes such as NOTCH2, EYA2, MACF1, TXNRD1, C17ORF28 and HECTD1 (Table S4). In agreement with these findings, Ingenuity Pathway Analysis (IPA) indicated a high score for networks composed of genes related to skeletal and muscular system development (Figure 2A), connective tissue disorders (Figure 2B) and cellular development (Figure $2 \mathrm{C}$ ), as well as other networks related to development (Table S5).

\section{Expression of candidate genes in another series of samples}

The expression of some genes (ADCY2, ANTXR1, CYR61, DNAH1, DUSP1, FGFR1, HECTD1, HNMT, KLF5, LOX, MACF1, MYH9, NOTCH2, SLC1A3, TACC1, TXNRD1 and USP16) was also evaluated by another methodology (RT-qPCR) in some samples not ana-

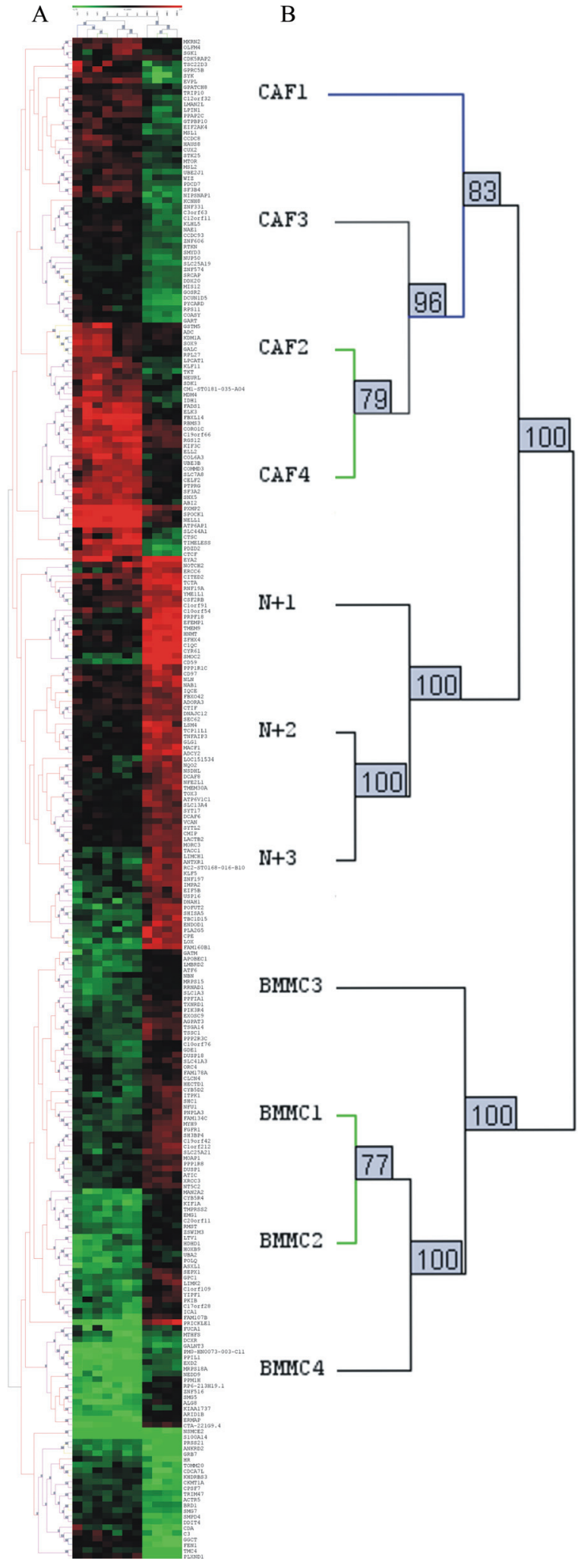

Figure 1 - Fibroblast analysis. (A) Fibroblast clustering according to cell origin. (B) Detail of the hierarchical clustering of fibroblasts from different origins based on the Euclidian distance of 267 genes differentially expressed in fibroblasts obtained from breast primary tumor (CAF), lymph node $(\mathrm{N}+)$ and bone marrow mesenchymal cells (BMMC). The reliability values were determined by the bootstrap technique and are shown in the gray boxes in the dendrogram. These genes correctly clustered the samples according to their origins. 

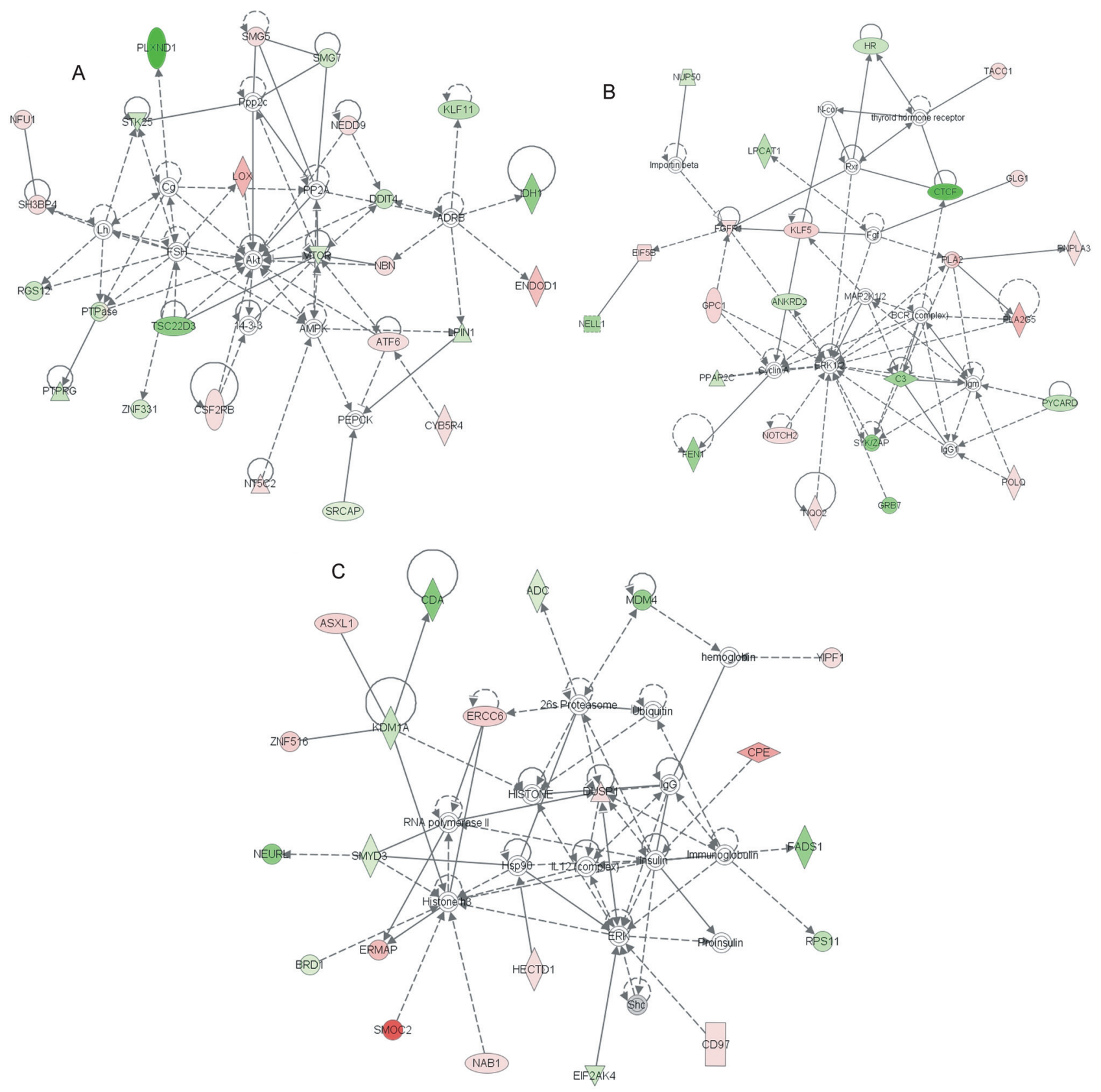

Figure 2 - Top networks observed in Ingenuity Pathway Analysis (IPA). (A) Network based on skeletal and muscular system development and function, cellular assembly and organization, cellular growth and proliferation. (B) Network based on connective tissue disorders, inflammatory disease, skeletal and muscular disorders. (C) Network based on cellular development, digestive system development and function, and embryonic development. The 267 genes differentially expressed among CAF, $\mathrm{N}+$ and $\mathrm{BMMC}$, as well as the fold change of CAF/BMMC, were used as input data for this analysis. Small circles identify proteins that act as hubs to interconnect groups of proteins to create protein clusters in pathways. Proteins in red were more expressed in bone marrow mesenchymal cells, whereas proteins in green were less expressed in bone marrow mesenchymal cells. Solid lines represent direct relationships. Lines connecting the proteins indicate known interrelationships obtained from the IPA database.

lyzed by microarray (biological validation set, indicated in Table 1). The selection of these genes was based on their relation to morphogenesis and differentiation, or on a foldchange in expression $>2$. In this analysis, $\mathrm{NOTCH} 2$ and SLC1A3 were significantly more expressed in fibroblasts from lymph nodes $(\mathrm{N}+)$ when compared to primary tumor samples (CAF) (Figure 3A); ADCY2 and SLC1A3 were confirmed more expressed in bone marrow (BMMC) than in primary tumor fibroblasts (CAF) (Figure 3B) and ADCY2 was significantly more expressed in bone marrow stromal cells (BMMC) when compared to lymph node fibroblasts $(\mathrm{N}+)$ (Figure 3C).

Since there was major agreement in the expression data obtained by microarray and RT-qPCR in the compari- 
son between primary tumor and bone marrow stromal cells, the expression of other genes was further tested in samples of the biological validation set from these tumor sites. This analysis confirmed that HECTD1, HNMT, LOX, MACF1 and USP16 were more expressed in bone marrow stromal cells (Figure 3D).

\section{Discussion}

The main goal of this work was to compare the gene expression profiles of stromal cells from the primary tumor, lymph node and bone marrow of breast cancer patients. In this analysis, 267 genes were differentially expressed among fibroblasts from primary tumor, lymph node and bone marrow. These genes represented $\sim 8 \%$ of the genes analyzed and appropriately discriminated among fibroblasts according to their origin. In contrast to small transcriptional differences between fibroblasts from the primary tumor and lymph nodes, a marked difference was observed when fibroblasts from these two sites were compared with bone marrow mesenchymal cells.

Transcriptional profile analysis has shown that fibroblasts from different anatomic sites and distinct donors exhibit topographic differentiation (Chang et al., 2002). Moreover, the fibroblast gene expression program follows a specific anatomic segmentation pattern that is homogeneous among samples, regardless of differences among host tissue donors (Rinn et al., 2006). These observations indicate that the positional memory of fibroblasts may be determined during embryogenesis. In the case of lymph node reticular cells, transcriptional differences were also observed in early passages, when compared to dermal fibroblasts; such differences can affect nearly $8 \%$ of analyzed genes (Vega et al., 2006). In addition, differences in the transcription profile of reticular cells from lymph nodes obtained from different organs have also been observed (Fletcher et al., 2011). In this case, topographic differences, which were greater than differences among tissue donors, probably involved the HOX gene family (Chang et al., 2002; Rinn et al., 2006).

Unexpectedly, only a small transcriptional difference ( $\sim 0.6 \%$ of the arrayed genes) was observed as differentially expressed between fibroblasts from the primary tumor and lymph node compared to a more striking difference between bone marrow stromal cells and fibroblasts from the other two sites. Similarity between the gene expression profiles of fibroblasts from these two sites could be explained by the presence of tumor cells that modulate their microenvironment in a similar way in the primary tumor and lymph node. Cancer cells are known to release growth factors, e.g., bFGF, VEGF, PDGF, EGF, TGFB and others, that modulate fibroblast functions such as proliferation and activation (Mueller and Fusenig, 2004; Kalluri and Zeisberg, 2006). In addition, malignant cells may markedly influence the CAF gene expression pattern (Rozenchan et al., 2009; Santos et al., 2011) and their metabolism (Pavlides et al., 2009; Sotgia et al., 2012).

However, this similarity may also be explained by the fibroblasts origin that involves a common source, including
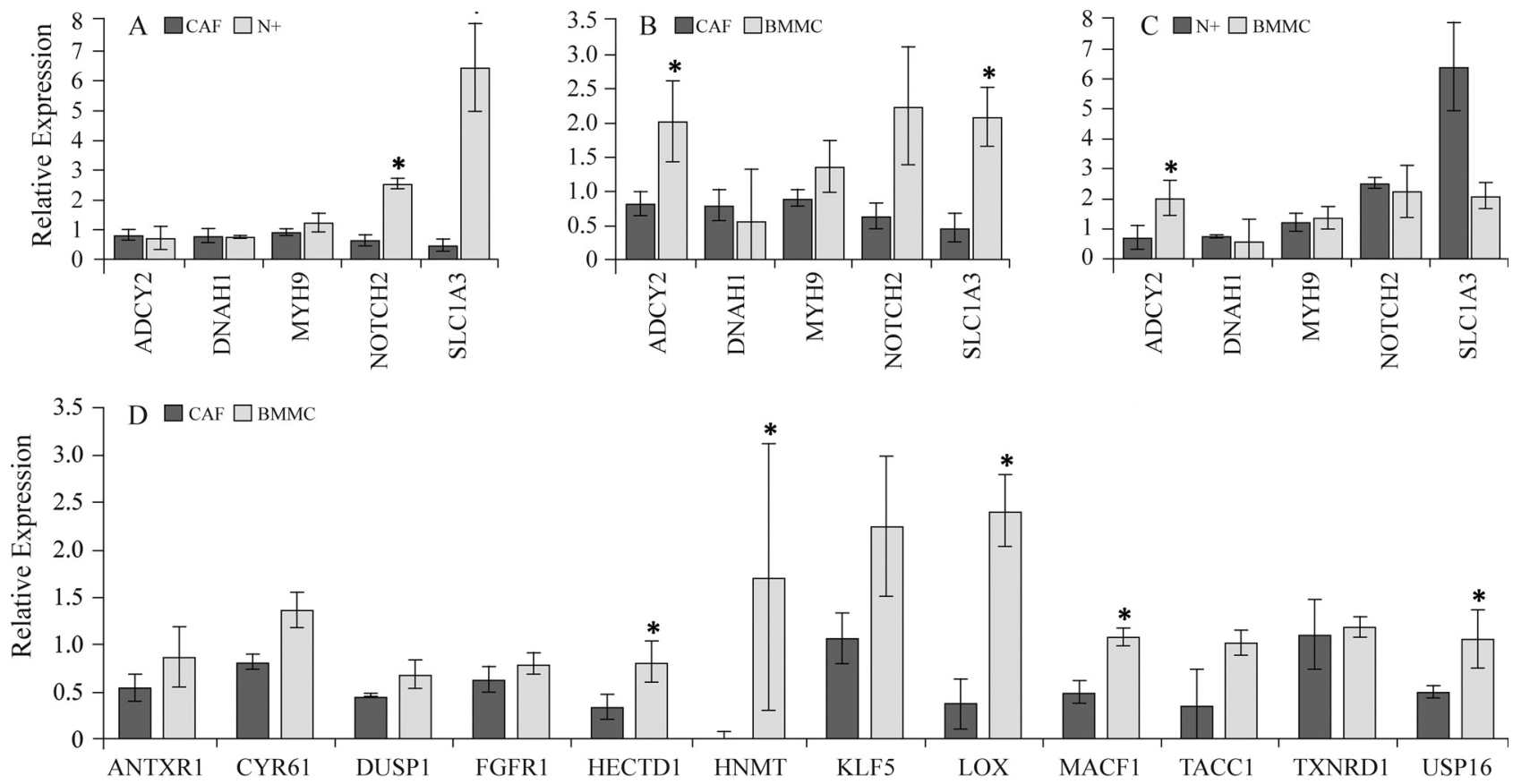

Figure 3 - Gene expression of fibroblast samples. (A) Primary tumor (CAF, $\mathrm{n}=6$ or 7$)$ vs. lymph node $(\mathrm{N}+, \mathrm{n}=3$ or 4$)$. (B) Primary tumor $(\mathrm{CAF}, \mathrm{n}=6$ or 7) $v$ s. bone marrow (BMMC, $\mathrm{n}=3$ or 4$)$. (C) Lymph node $(\mathrm{N}+, \mathrm{n}=3$ or 4$) v s$. bone marrow $(\mathrm{BMMC}, \mathrm{n}=3$ or 4$)$. (D) Primary tumor $(\mathrm{CAF}, \mathrm{n}=3) v s$. bone marrow $(\mathrm{BMMC}, \mathrm{n}=3)$. Relative expression based on $2^{-\Delta \Delta \mathrm{Ct}}$. PPIA was used as a reference gene, and average $\mathrm{Ct}$ values for the same transcript were used as the sample reference. Bars represent mean $\pm \mathrm{SE} . * \mathrm{p} \leq 0.05$ (Mann-Whitney test). 
the epithelial-mesenchymal transition, the co-migration of CAFs and cancer cells to metastatic sites, and the migration and differentiation of mesenchymal stem cells from the bone marrow to tumor sites. None of these is necessarily exclusive, but may occur simultaneously, in different proportions, during tumor-stroma co-evolution (Hanahan and Weinberg, 2011).

Up to $20 \%$ of tumor-associated fibroblasts may originate from bone marrow mesenchymal stem cells (Quante $e t$ al., 2011). By using a mouse model of gastric cancer and the tracking of bone marrow mesenchymal stem cells with a reporter gene that allows their discrimination from resident cells in the tumor microenvironment, it was shown that only myofibroblasts derived from bone marrow mesenchymal stem cells could survive more than four passages in culture; resident myofibroblasts could not survive beyond the fourth passage (Quante et al., 2011). In addition, conditioned media from cancer cells can influence the differentiation of mesenchymal stem cells into CAFs (Mishra et al., 2008).

In agreement with these findings, in the present work the expression of some genes involved in mesoderm formation, response to wounding, regeneration and embryonic morphogenesis was enhanced in bone marrow mesenchymal cells when compared with $\mathrm{CAF}$ or $\mathrm{N}+$. These genes include HECTD1 (HECT domain containing E3 ubiquitin protein ligase 1), involved in negative regulation of the Wnt-Axin pathway (Tran et al., 2013), MACF1 (microtubule-actin crosslinking factor 1) involved in the regulation of cell migration after ErbB2 stimulation (Benseddik et al., 2013), LOX (lysyl oxidase) involved in creating a fibrotic environment favorable to tumor cell colonization (Cox et al., 2013) and SLC1A3 (solute carrier family 1 [glial high affinity glutamate transporter], member 3) involved in the response to wounding. The genes ADCY2 (adenylate cyclase 2 [brain]) involved in signal transduction from membrane receptors, HNMT (histamine Nmethyltransferase) involved in histamine metabolism and USP16 (ubiquitin specific peptidase 16) involved in histone deubiquitination and cell cycle were also found to be more expressed in bone marrow mesenchymal cells.

This work represents the first attempt to characterize the transcriptional profiles of fibroblasts from different sites in breast cancer patients, including primary cultures of stromal cells from bone marrow and lymph node, which are very difficult to establish and maintain in culture. The majority of studies that have investigated the origin of cancer fibroblasts have used mice (Quante et al., 2011; Kidd et al., 2012) and their findings are sometimes difficult to translate to humans. On the other hand, studies that have examined the differentiation of bone marrow mesenchymal cells to CAFs have used mainly bone marrow from health donors and have not considered the influence of the tumor on this site (Mishra et al., 2008). One of the shortcomings of the present study was the incomplete agreement between the expression data obtained by microarray and RT-qPCR. One possible explanation for this discrepancy is that fibroblast primary cultures change their transcriptional pattern over time, especially lymph node stromal cells (Vega et al., 2006), and the RT-qPCR expression data were obtained from fibroblasts in later passages than those used for the microarray. Although unpaired samples were used in this work, it was previously shown that samples obtained from different sites of the same individual, or subjected to differential experimental conditions, may cluster together $(50 \%$ of the samples) or in different branches (another 50\%) (Folgueira et al., 2005; Perou et al., 2000), thereby blurring transcriptional differences attributed to tissue specificities. In the present work, the analysis of unpaired tissue samples allowed the identification of differentially expressed genes that were homogeneously expressed within the groups.

In summary, only small differences were observed in the transcriptional profiles of fibroblasts from the primary tumor and lymph node of breast cancer patients. In contrast, the difference in gene expression between these two tissues and bone marrow stromal cells was much greater. The differentially expressed genes identified here may reflect a distinct differentiation profile or adaptations that bone marrow mesenchymal cells may undergo to become CAFs. However, functional studies are needed to confirm the influence of these genes in the differentiation of bone marrow mesenchymal cells into CAFs.

\section{Acknowledgments}

The authors thank Cristina Piñeiro Grandal for editing the figures. This work was supported by Fundação de Amparo à Pesquisa do Estado de São Paulo (FAPESP), Coordenação de Aperfeiçoamento de Pessoal de Nível Superior (CAPES) and Conselho Nacional de Desenvolvimento Científico e Tecnológico (CNPq).

\section{References}

Baugh LR, Hill AA, Brown EL and Hunter CP (2001) Quantitative analysis of mRNA amplification by in vitro transcription. Nucleic Acids Res 29:E29.

Benseddik K, Sen Nkwe N, Daou P, Verdier-Pinard P and Badache A (2013) ErbB2-dependent chemotaxis requires microtubule capture and stabilization coordinated by distinct signaling pathways. PLoS One 8:e55211.

Brentani RR, Carraro DM, Verjovski-Almeida S, Reis EM, Neves EJ, de Souza SJ, Carvalho AF, Brentani H and Reis LF (2005) Gene expression arrays in cancer research: Methods and applications. Crit Rev Oncol Hematol 54:95-105.

Campos LT, Brentani H, Roela RA, Katayama ML, Lima L, Rolim CF, Milani C, Folgueira MA and Brentani MM (2013) Differences in transcriptional effects of $1 \alpha, 25$ dihydroxyvitamin D3 on fibroblasts associated to breast carcinomas and from paired normal breast tissues. J Steroid Biochem Mol Biol 133:12-24.

Chang HY, Chi JT, Dudoit S, Bondre C, van de Rijn M, Botstein D and Brown PO (2002) Diversity, topographic differentia- 
tion, and positional memory in human fibroblasts. Proc Natl Acad Sci USA 99:12877-12882.

Cox TR, Bird D, Baker AM, Barker HE, Ho MW, Lang G and Erler JT (2013) LOX-mediated collagen crosslinking is responsible for fibrosis-enhanced metastasis. Cancer Res 73:1721-1732.

de Kruijf EM, van Nes JG, van de Velde CJ, Putter H, Smit VT, Liefers GJ, Kuppen PJ, Tollenaar RA and Mesker WE (2011) Tumor-stroma ratio in the primary tumor is a prognostic factor in early breast cancer patients, especially in triple-negative carcinoma patients. Breast Cancer Res Treat 125:687-696.

Duda DG, Duyverman AM, Kohno M, Snuderl M, Steller EJ, Fukumura D and Jain RK (2010) Malignant cells facilitate lung metastasis by bringing their own soil. Proc Natl Acad Sci USA 107:21677-21682.

Edge SB and Compton CC (2010) The American Joint Committee on Cancer: The 7th edition of the AJCC cancer staging manual and the future of TNM. Ann Surg Oncol 17:1471-1474.

El-Haibi CP and Karnoub AE (2010) Mesenchymal stem cells in the pathogenesis and therapy of breast cancer. J Mammary Gland Biol Neoplasia 15:399-409.

Fletcher AL, Malhotra D, Acton SE, Lukacs-Kornek V, Bellemare-Pelletier A, Curry M, Armant M and Turley SJ (2011) Reproducible isolation of lymph node stromal cells reveals site-dependent differences in fibroblastic reticular cells. Front Immunol 2:35.

Folgueira MA, Carraro DM, Brentani H, Patrão DF, Barbosa EM, Netto MM, Caldeira JR, Katayama ML, Soares FA, Oliveira CT, et al. (2005) Gene expression profile associated with response to doxorubicin-based therapy in breast cancer. Clin Cancer Res 11:7434-7443.

Guarino M, Tosoni A and Nebuloni M (2009) Direct contribution of epithelium to organ fibrosis: Epithelial-mesenchymal transition. Hum Pathol 40:1365-1376.

Hanahan D and Weinberg RA (2011) Hallmarks of cancer: The next generation. Cell 144:646-674.

Kalluri R and Zeisberg M (2006) Fibroblasts in cancer. Nat Rev Cancer 6:392-401.

Karnoub AE, Dash AB, Vo AP, Sullivan A, Brooks MW, Bell GW, Richardson AL, Polyak K, Tubo R and Weinberg RA (2007) Mesenchymal stem cells within tumour stroma promote breast cancer metastasis. Nature 449:557-563.

Kidd S, Spaeth E, Watson K, Burks J, Lu H, Klopp A, Andreeff M and Marini FC (2012) Origins of the tumor microenvironment: Quantitative assessment of adipose-derived and bone marrow-derived stroma. PLoS One 7:e30563.

Kim SI, Park BW and Lee KS (2006) Comparison of stagespecific outcome of breast cancer based on 5th and 6th AJCC staging system. J Surg Oncol 93:221-227.

Livak KJ and Schmittgen TD (2001) Analysis of relative gene expression data using real-time quantitative PCR and the $\left.2{ }^{-\triangle \Delta C T}\right)$ method. Methods 25:402-408.

Martinez LM, Vallone VB, Labovsky V, Choi H, Hofer EL, Feldman L, Bordenave RH, Batagelj E, Dimase F, Villafane $\mathrm{AR}$, et al. (2013) Changes in the peripheral blood and bone marrow from untreated advanced breast cancer patients that are associated with the establishment of bone metastases. Clin Exp Metastasis 31:213-232.

Martinez-Outschoorn UE, Lisanti MP and Sotgia F (2014) Catabolic cancer-associated fibroblasts transfer energy and bio- mass to anabolic cancer cells, fueling tumor growth. Semin Cancer Biol 25C:47-60.

Mishra PJ, Humeniuk R, Medina DJ, Alexe G, Mesirov JP, Ganesan S, Glod JW and Banerjee D (2008) Carcinoma-associated fibroblast-like differentiation of human mesenchymal stem cells. Cancer Res 68:4331-4339.

Mueller MM and Fusenig NE (2004) Friends or foes - Bipolar effects of the tumour stroma in cancer. Nat Rev Cancer 4:839849.

Orimo A, Gupta PB, Sgroi DC, Arenzana-Seisdedos F, Delaunay T, Naeem R, Carey VJ, Richardson AL and Weinberg RA (2005) Stromal fibroblasts present in invasive human breast carcinomas promote tumor growth and angiogenesis through elevated SDF-1/CXCL12 secretion. Cell 121:335348.

Patocs A, Zhang L, Xu Y, Weber F, Caldes T, Mutter GL, Platzer P and Eng C (2007) Breast-cancer stromal cells with TP53 mutations and nodal metastases. N Engl J Med 357:25432551.

Paulsson J and Micke P (2014) Prognostic relevance of cancer-associated fibroblasts in human cancer. Semin Cancer Biol 25C:61-68.

Pavlides S, Whitaker-Menezes D, Castello-Cros R, Flomenberg N, Witkiewicz AK, Frank PG, Casimiro MC, Wang C, Fortina P, Addya S, et al. (2009) The reverse Warburg effect: Aerobic glycolysis in cancer associated fibroblasts and the tumor stroma. Cell Cycle 8:3984-4001.

Perou CM, Sorlie T, Eisen MB, van de Rijn M, Jeffrey SS, Rees CA, Pollack JR, Ross DT, Johnsen H, Akslen LA, et al. (2000) Molecular portraits of human breast tumours. Nature 406:747-752.

Petersen OW, Nielsen HL, Gudjonsson T, Villadsen R, Rank F, Niebuhr E, Bissell MJ and Ronnov-Jessen L (2003) Epithelial to mesenchymal transition in human breast cancer can provide a nonmalignant stroma. Am J Pathol 162:391-402.

Quante M, Tu SP, Tomita H, Gonda T, Wang SS, Takashi S, Baik GH, Shibata W, Diprete B, Betz KS, et al. (2011) Bone marrow-derived myofibroblasts contribute to the mesenchymal stem cell niche and promote tumor growth. Cancer Cell 19:257-272.

Rinn JL, Bondre C, Gladstone HB, Brown PO and Chang HY (2006) Anatomic demarcation by positional variation in fibroblast gene expression programs. PLoS Genet 2:e119.

Rozenchan PB, Carraro DM, Brentani H, de Carvalho Mota LD, Bastos EP, Ferreira EN, Torres CH, Katayama ML, Roela RA, Lyra EC, et al. (2009) Reciprocal changes in gene expression profiles of cocultured breast epithelial cells and primary fibroblasts. Int J Cancer 125:2767-2777.

Santos RP, Benvenuti TT, Honda ST, Del Valle PR, Katayama ML, Brentani HP, Carraro DM, Rozenchan PB, Brentani MM, de Lyra EC, et al. (2011) Influence of the interaction between nodal fibroblast and breast cancer cells on gene expression. Tumour Biol 32:145-157.

Sekiya I, Larson BL, Smith JR, Pochampally R, Cui JG and Prockop DJ (2002) Expansion of human adult stem cells from bone marrow stroma: Conditions that maximize the yields of early progenitors and evaluate their quality. Stem Cells 20:530-541.

Shimoda M, Mellody KT and Orimo A (2010) Carcinoma-associated fibroblasts are a rate-limiting determinant for tumour progression. Semin Cell Dev Biol 21:19-25. 
Sotgia F, Whitaker-Menezes D, Martinez-Outschoorn UE, Flomenberg N, Birbe RC, Witkiewicz AK, Howell A, Philp NJ, Pestell RG and Lisanti MP (2012) Mitochondrial metabolism in cancer metastasis: Visualizing tumor cell mitochondria and the "reverse Warburg effect" in positive lymph node tissue. Cell Cycle 11:1445-1454.

Surowiak P, Murawa D, Materna V, Maciejczyk A, Pudelko M, Ciesla S, Breborowicz J, Murawa P, Zabel M, Dietel M, et al. (2007) Occurence of stromal myofibroblasts in the invasive ductal breast cancer tissue is an unfavourable prognostic factor. Anticancer Res 27:2917-2924.

Tran H, Bustos D, Yeh R, Rubinfeld B, Lam C, Shriver S, Zilberleyb I, Lee MW, Phu L, Sarkar AA, et al. (2013) HectD1 E3 ligase modifies adenomatous polyposis coli (APC) with polyubiquitin to promote the APC-axin interaction. J Biol Chem 288:3753-3767.

Trimboli AJ, Cantemir-Stone CZ, Li F, Wallace JA, Merchant A, Creasap N, Thompson JC, Caserta E, Wang H, Chong JL, et al. (2009) Pten in stromal fibroblasts suppresses mammary epithelial tumours. Nature 461:1084-1091.

Vega F, Coombes KR, Thomazy VA, Patel K, Lang W and Jones D (2006) Tissue-specific function of lymph node fibroblastic reticulum cells. Pathobiology 73:71-81.

Wang E, Miller LD, Ohnmacht GA, Liu ET and Marincola FM (2000) High-fidelity mRNA amplification for gene profiling. Nat Biotechnol 18:457-459.

Yeung TM, Buskens C, Wang LM, Mortensen NJ and Bodmer WF (2013) Myofibroblast activation in colorectal cancer lymph node metastases. Br J Cancer 108:2106-2115.

Zeisberg EM, Potenta S, Xie L, Zeisberg M and Kalluri R (2007) Discovery of endothelial to mesenchymal transition as a source for carcinoma-associated fibroblasts. Cancer Res 67:10123-10128.

\section{Internet Resources}

Primer Blast, http://www.ncbi.nlm.nih.gov/tools/primer-blast.

DAVID (Database for Annotation, Visualization and Integrated Discovery) v.6.7, http://david.abcc.ncifcrf.gov/tools.jsp (access date - Sept 14, 2013).

\section{Supplementary Material}

The following online material is available for this article:

Table S1 - Primers used in this work.

Table S2 - Differentially expressed genes.

Table S3 - Functional annotation of biological processes of less expressed genes in stromal cells derived from bone marrow.

Table S4 - Functional annotation of biological processes of more expressed genes in stromal cells derived from bone marrow.

Table S5 - Top networks observed in Ingenuity Pathway Analysis (IPA).

Figure S1 - Heat map of differentially expressed genes that gave no signal.

This material is available as part of the online article from http://www.scielo.br/gmb.

Associate Editor: Jeremy A. Squire

License information: This is an open-access article distributed under the terms of the Creative Commons Attribution License, which permits unrestricted use, distribution, and reproduction in any medium, provided the original work is properly cited. 